\title{
Supramolecular Organic Nanofibers with Highly Efficient and Stable Visible Light Photooxidation Performance
}

Jun Wang, Wen Shi, Di Liu, ZiJian Zhang,Yongfa Zhu* and Dong Wang* Department of Chemistry, Tsinghua University, Beijing 100084, P.R. China

Corresponding author: E-mail: zhuyf@tsinghua.edu.cn; Fax: +86-10-62787601; Tel: $+86-10-62787601$. 
Abstract: The development of organic materials for visible light driven photocatalytic is regarded as one of the most promising avenues to solve environment and solar-energy utilization issue. Here, we present that one-dimensional supramolecular organic nanofibers, self-assembled by a carboxy-substituent perylene diimide (PDI) molecule through H-type $\pi-\pi$ stacking and hydrogen bonding, can act as a robust and effective photocatalyst for both organic pollutants degradation and water oxidation under visible light without the apparent need for an added metal co-catalysts. We corroborate that the highly efficient and stable activity of such supramolecar photocatalyst are attributed to the introduction of terminal carboxyl group, which leads to well-defined and stable $\mathrm{H}$ type $\pi-\pi$ stacking, and constructs the internal electric field in supramolecular nanofibers, thereby resulting in the deepening of valence band (VB) and the enhancement of migration and separation efficiency of photo-induced charge carriers. Our findings may help the development of semiconducting-based organic supramolecular materials for applications in environment protection and water splitting.

Keywords: One-dimensional Supramolecular photocatalyst, H-type $\pi-\pi$ stacking, Visible light, Oxygen evolution, High stability 


\section{Introduction}

The development of organic materials for water splitting under visible-light irradiation was regarded as one of the most promising scenarios for solar-energy utilization.[1] Over the past several years, the search for such materials has focused mainly on the metal-organic frameworks[2] and covalent organic polymers,[3-6] such as polymeric carbon nitride,[3] poly (diphenylbutadiyne)[4] and poly(azomethines)[6]. However, the present progress of research for these organocatalysts was far from the practical application owing to their intrinsic disadvantages of low sustainability, high cost, potential toxicity, complex synthetic route, poor structure tunability and limited photo-activity. Moreover, these organic photocatalysts were always employed to reduce water rather than act as a water oxidant. It is well known that the bottleneck reaction of water splitting was oxygen evolution due to the large overpotential and slow kinetics of four-electron charge-transfer process.[7] Therefore, the search for active organic photocatalysts that directly split water under visible light remains one of the most challenging tasks for solar-energy utilization.

PDI (perylene-3,4,9,10-tetracarboxylic diimide) and its derivatives were regarded as one of the best n-type organic semiconductors and have been widely applied in phosphors,[8] switches,[9] sensors,[10] organic field-effect transistors (OFETs) [11, 12] and solar cells $[13,14]$ owing to its unique optical and electronic properties. 15 The high thermal and photostability of PDI makes it an ideal candidate for water oxidation photocatalyst. Recently, the supramolecular architectures of PDI were employed as antennae chromophores working with the photocatalysts to construct water splitting system,[16-19] such as PDI-Ni,[16] PDI-Ru,[17] PDI-TiO ${ }_{2}$ [18] and PDI-C $3 \mathrm{~N}_{4}$ [19]. However, in these composite systems, PDIs were introduced to act as dye-sensitizer rather than photocatalyst. Very recent, our group demonstrated that the purely unmodified perylene diimide (PTCDI) supramolecular nonoassemblies can work as central catalyst to harness visible light to decompose organic pollutants as well as oxide water.[20] This result offers a new vista for the development of supramolecular organic materials in water splitting and waste-water cleaning. However, the photo-activities of such PTCDI nonoassemblies were still poor, especially for oxygen evolution (only $2.6 \mu \mathrm{mol} \mathrm{h} \mathrm{h}^{-1} \mathrm{~g}^{-1}$. Moreover, limited by its intrinsic insolubility in organic solvents,[15] 
the supramolecular self-assembly of PTCDI can be only performed in few special solvents such as concentrated sulfuric acid or m-cresol,[21] which great restrict its structure tunability and photocatalytic performance.

Herein, we demonstrate that supramolecular nanofibres of a perylene diimide (PDI) derivative, $N, N^{\prime}$-di(propanoic acid)-perylene-3,4,9,10-tetracarboxylic diimide (Scheme S1a),[22] are highly efficient and stable under visible light for photooxidation. The phenol degradation and oxygen evolution rate of such supramolecular photocatalyst are an order of magnitude higher than that of unmodified perylene diimide (PTCDI) and g$\mathrm{C}_{3} \mathrm{~N}_{4}$. The photocatalytic activities, morphology and structure of these supramolecular nanofibers remain unchanged after repeated cycles in acid condition. The introduction of terminal carboxyl group at the imides of PDI leads to well-defined and stable H-type $\pi-\pi$ stacking, and constructs the internal electric field in supramolecular nanofibers, which are attributed to the highly efficient and stable activity of such supramolecar photocatalyst. Furthermore, the effect of intermolecular packing arrangement on supramoleclar electronic energy level structure and photocatalytic performance are investigated by incorporating theoretical and experimental methods.

\section{Experimental Section}

\subsection{Preparation of PDI nanofibers}

The perylene-3,4,9,10-tetracarboxylic dianhydride, perylene-3,4,9,10tetracarboxylic diimide, 3-aminopropanoic acid, imidazole, n-Propylaminwe were supplied by Aldrich. Dicyandiamide and silver nitrate were purchased from Sinopharm Chemical Reagent Corp, P. R. China. All reagents were analytically pure and used without further purification.

$200.0 \mathrm{~mL}$ stock solution of $\mathrm{N}, \quad \mathrm{N}$ '-di(propanoic acid)-perylene-3,4,9,10tetracarboxylic diimide $(5.0 \mathrm{mM})$ was prepared with addition of $834 \mu \mathrm{L}$ of TEA. The solution thus prepared looked clear, in red colour. PDI nanofibers were formed by adding $27.3 \mathrm{ml} 4.0 \mathrm{M} \mathrm{HCl}$. It was washed thoroughly with distilled water until the $\mathrm{pH}$ of washings turned to be neutral. The collected solid was dried in at $60{ }^{\circ} \mathrm{C}$.

\subsection{Characterizations}

UV-vis spectra of PDI were recorded by Hitachi U-3010 UV-vis spectrophotometer. 
Morphologies and structures of the prepared samples were examined by JEM 1200 transmission electron microscopy (TEM) at an accelerating voltage of $100 \mathrm{kV}$. Atomic force microscopy (AFM) images were recorded by using a SPM-9700 scanning probe microscope (Shimadzu Corporation). UV-vis diffuse reflection spectra (DRS) were obtained on a Hitachi U-3010 UV-vis spectrophotometer. $\mathrm{BaSO}_{4}$ was used as the reflectance standard in the experiment. X-ray photoelectron spectroscopy (XPS ULVAC-PHI, Quantera) was used to estimate the VB position of solid PDI SNW. The charge effect was calibrated using the binding energy of $\mathrm{C} 1 \mathrm{~s}$.

The electrochemical and photoelectrochemical were measured on an electrochemical system (CHI-660B, China). The photoelectric responses of the photocatalysts as light on and off were measured at $0.0 \mathrm{~V}$. A standard three electrode cell with ITO or Pt as a working electrode, a platinum disk as a counter electrode, and a standard calomel electrode (SCE) as reference electrode were used in photoelectric studies. $0.1 \mathrm{M} \mathrm{Na}_{2} \mathrm{SO}_{4}$ was used as the electrolyte solution. Potentials were given with reference to the SCE. Differential pulse voltammograms (DPV) was used to estimate the HOMO/LUMO levels of PDI monomer. The concentration of PDI monomer was $1 \times 10^{-5} \mathrm{M}$. The supporting electrolyte was $0.1 \mathrm{M} \mathrm{NH}_{4} \mathrm{PF}_{6}$. The scan rate was $100 \mathrm{mV} \mathrm{s}^{-1}$.

\subsection{Computational methodology}

In order to calculate the absolute energy level position of HOMO and LUMO of $\pi-\pi$ stacked PDI aggregates (comprising $\mathrm{n}=1,2,3$ and infinite monomers), vacuum energy level calibration method was applied. A one-dimensional stack model was constructed. The vacuum energy level in the stack is zero. The ionic positions of the starting structures in the stack were optimized by the projector augmented wave (PAW) method with the Perdew-Burke-Ernzerhof including dispersion (PBE-D) exchange correlation functional in the Vienna ab initio simulation package (VASP, version 5.3.2).[23, 24] Throughout the calculations, the convergence criterion of the total energy was set to $10^{-5} \mathrm{eV}$ in the self-consistent field iteration. The cutoff energy for the plane-wave basis set was set to $600 \mathrm{eV}$. The $\mathrm{k}$-mesh of $1 \times 1 \times 1$ was used for monomer, dimer and trimer; and that of $1 \times 1 \times 4$ was applied for the infinite stacked PDI aggregation. The cutoff radius for pair interactions was set to be $50 \AA$. 


\subsection{Photocatalytic experiments}

The photocatalyst powders $(50 \mathrm{mg})$ were dispersed by a magnetic stirrer in $100 \mathrm{ml}$ of $\mathrm{AgNO}_{3}(\mathrm{aq})$ in a reaction cell. The visible light source was obtained by a $300 \mathrm{~W}$ xenon lamp with cutoff filter $(>420 \mathrm{~nm})$ or bandpass filter $(500,550 \ldots 700 \pm 15 \mathrm{~nm})$. The irradiance was measured to be $152 \mathrm{~mW} / \mathrm{cm}^{2}$ (CEL-NP2000). The amount of evolved oxygen was determined using a gas chromatograph (GC7920, TCD, Ar carrier) For quantum efficiency measurements, bandpass filter $(600 \pm 15 \mathrm{~nm})$ was used with the irradiance was measured to be $48 \mathrm{~mW} / \mathrm{cm}^{2}$. The quantum yield was calculated from the percentage of the number of reacted electrons during $\mathrm{O}_{2}$ evolution to the number of incident photons.

The photodegradation reactions were carried out in quartz tube reactor with a $50 \mathrm{ml}$ $5 \mathrm{ppm}$ phenol solution and $25 \mathrm{mg}$ photocatalyst powders. The $\mathrm{pH}$ of solution was adjusted by $0.1 \mathrm{M} \mathrm{HCl}$ or $0.1 \mathrm{M} \mathrm{NaOH}$. The visible light source was obtained by a 500 W xenon lamp with cutoff filter $(>420 \mathrm{~nm})$. Before irradiation, the suspension solution was first ultrasonic dispersed in dark for $15 \mathrm{~min}$ and then magnetically stirred for $1 \mathrm{~h}$ to reach the adsorption-desorption equilibrium. At given time intervals, $2.5 \mathrm{~mL}$ solution were sampled and centrifuged to remove the photocatalysts. The concentration of phenol characterized using HPLC (Lumtech) system. Venusil XBP-C18 (250 mm $\times 4.6$ mm i.d., $5 \mu \mathrm{m}$ ) reversed phase column was used. The degradation process was fitted to pseudo-first-order kinetics, and the value of the rate constant $k$ was equal to the corresponding slope of the fitting line.

\section{Results and Discussion}

\subsection{Morphology and $\pi-\pi$ stacking structure of PDI nanofibers}

The morphologies of as-prepared PDI supramolecular building blocks were investigated by SEM, TEM and AFM observations. The untreated PDI sample exhibits a morphology of irregular microrod (denoted as bulk PDI, Fig. 1a and S1). The supramolecular PDI nanofibers (denoted as nano PDI) were prepared via a pH triggered hydrogelation method developed by Zang et al (Scheme S1b).[22] Typically, bulk PDI was first dissolved by the solution of trimethylamine (TEA), which resulted in homogeneous solution of PDI with clear red colour. And then red brown gel of PDI was 
separated out after dropwise adding the $\mathrm{HCl}$. After regrowth, well-defined nanofibers were formed only by $\pi-\pi$ stacking and hydrogen bonding (Fig. 1c) with a diameter of $\sim 20-50 \mathrm{~nm}$ and a length of $\sim 0.8-1.2 \mu \mathrm{m}$ (Fig. $1 \mathrm{~b}$ and S2). The height profile of atomic force microscopy (AFM, Fig. S3) confirms that the diameter of a single nanofiber was about $27 \mathrm{~nm}$. It is well known that the mean free path of organic materials $(\sim 10 \mathrm{~nm})$ was shorter than that of inorganic materials $(\sim 100 \mathrm{~nm})$. Therefore, the nanoscale of these supramolecular building blocks was important to the migration efficiency of photo-induced charge carriers. Moreover, the specific surface area (BET) of nano PDI was $41.62 \mathrm{~m}^{2} / \mathrm{g}$ with a bore diameter about $20 \mathrm{~nm}$ (Fig. S4), which was much larger than that of bulk PDI (Table S1). Small size and large specific surface area of PDI nanofibers are benefit to its photocatalytic activity.

The introduction of short carboxylic acid side chains not only enables sufficient water solubility, but also facilitates the columnar staking between the perylene backbones. The strong $\pi-\pi$ stacking interactions of PDI molecules in self-assembled supramolecular can be revealed from UV-vis absorption spectra, X-ray diffraction (XRD) and micro-Raman spectra. The UV-vis absorption spectra of PDI monomer and PDI nanofiber gel were shown in Fig. 1c. The absorption spectrum of PDI dissolved in $\mathrm{H}_{2} \mathrm{O} / \mathrm{CH}_{3} \mathrm{OH}(\mathrm{v} / \mathrm{v}=1: 1$, Scheme $\mathrm{S} 1 \mathrm{~b})$ shows three pronounced peaks in the range of $400-550 \mathrm{~nm}$, which correspond to the $0-0,0-1$ and $0-2$ electronic transitions of monomeric PDI molecules, respectively (black dotted curve).[15] After self-assembly, the maximum absorption has a great blue-shift and the fine curve structure of absorption spectrum was lost, indicating the strong H-type $\pi$ - $\pi$ stacking between the PDI skeletons in supramolecular nanofibers (red curve).[25]

Fig. 1d shows the solid XRD patterns of bulk PDI and nano PDI samples. No significantly changes in crystal structure can be observed. However, the intensity of diffraction decreased obviously after regrowth because of the nanoctystalline of PDI supramolecular block. The typical d-spacing of $\pi$ - $\pi$ stacking was $\sim 3.34-3.55 \AA$,[22] corresponding to the P1 peak in the XRD patterns. It is clear that the intensity of P1 peak was in the bulk PDI sample lower than that of P0 peak. On the contrary, the relative intensity of $\mathrm{P} 1$ to $\mathrm{P} 0\left(\mathrm{~A}_{\mathrm{P} 1} / \mathrm{A}_{\mathrm{P} 0}>1\right)$ in nano $\mathrm{PDI}$ was reversal of that of bulk $\mathrm{PDI}\left(\mathrm{A}_{\mathrm{P} 1} / \mathrm{A}_{\mathrm{P} 0}\right.$ $<1)$. The increase of relative intensity of P1 peak in PDI nanofibers indicates that higher 
order $\pi-\pi$ stacking was formed after regrowth. Moreover, the typical XRD peak (P1) of $\pi-\pi$ stacking for nano PDI appeared at a higher angle. Such an observation indicates that $d$-spacing of $\pi-\pi$ stacking in nano PDI $(0.34 \mathrm{~nm})$ was smaller than that of bulk PDI. Higher order $\pi-\pi$ stacking and smaller $d$-spacing of $\pi$ - $\pi$ stacking results in long-range electrons delocalization the high density of electron cloud overlap, which was benefit to the migration of charge carriers.

The Raman spectra of bulk PDI and nano PDI samples exhibit several Raman modes with frequencies of $1318 \mathrm{~cm}^{-1}, 1394 \mathrm{~cm}^{-1}, 1471 \mathrm{~cm}^{-1}, 1589 \mathrm{~cm}^{-1}, 1602 \mathrm{~cm}^{-1}$ (Fig. S5). No significantly changes in Raman frequency can be observed, indicating the crystal phase of PDI was stay during regrowth. The $1602 \mathrm{~cm}^{-1}$ Raman active normal mode corresponds to the shrinking of the perylene aromatic ring core coupled to the $\mathrm{C}=\mathrm{O}$ antisymmetric and the stretching of the in-plane $\mathrm{C}=\mathrm{C} / \mathrm{C}-\mathrm{C}$, which was sensitive to the $\pi-\pi$ stacking intermolecular interactions.[14] In contrast, Raman mode of $\mathrm{CH}$ in plane bending at $1318 \mathrm{~cm}^{-1}$ was insensitive to the $\pi-\pi$ stacking intermolecular interactions.[14] Therefore, we can utilize the $1602 \mathrm{~cm}^{-1} / 1318 \mathrm{~cm}^{-1}$ Raman intensity ratio as an effective marker to monitor the degree of $\pi-\pi$ stacking in PDI.[26] The relative intensity of 1602 $\mathrm{cm}^{-1}$ to $1318 \mathrm{~cm}^{-1}$ in nano PDI sample was about 0.67 , which was higher than that of bulk PDI (0.44), indicating higher order $\pi-\pi$ stacking in nano PDI.

Fig. 1f shows the light absorption characters of bulk PDI and nano PDI samples. Both bulk PDI and nano PDI can absorb solar energy with a wavelength shorter than $\sim 650 \mathrm{~nm}$, which virtually cover full spectrum of visible light. Compared to bulk PDI, the nano PDI sample exhibits a higher absorbance and wider spectrum response. The absorption edges of bulk PDI and nano PDI were about $712 \mathrm{~nm}$ and $734 \mathrm{~nm}$, corresponding to the bandgaps about $1.74 \mathrm{eV}$ and $1.69 \mathrm{eV}$. Generally, the nanoctystalline of materials always lead to blue shift of absorption edge, whereas the nanoctystalline of PDI supramolecular block leads to a red shift of absorption edge in our research. Such abnormal phenomenon in our study can be attributed to the narrowing of bandgap caused by $\pi$-conjugated system, which will be further discussed as follows.

\subsection{Electronic properties of PDI nanofibers}


The top of Fig. 2a shows the calculational frontier orbitals of PDI molecule. It was found that both LUMO and HOMO levels of PDI molecule consist of carbon and oxygen elements only from perylene core. The two nitrogen positions at the imides of perylene core were nodes in the $\pi$-orbital wave function.[27] In other word, the intrinsic electronic property of PDI cannot be affect by side chains substituent groups.[28] Since the side chains have no significant effect on the electronic property of PDI molecule, the electronic structure of PDI supramolecular blocks was mainly dependent on the $\pi$ $\pi$ stacking.[29] Here, the influence of H-type $\pi-\pi$ stacking on the electronic structure of PDI nanofibers were investigated combined with theoretical and experimental methods. For theoretical study, ab initio density functional theory (DFT) calculations have been carried out. In view of the fact that accurate stacking model of PDI nanofibers cannot be established due to the absence of crystallographic parameters, an ideal model of quasi-one-dimensional H-type $\pi-\pi$ stack was employed to investigate the effects of $\mathrm{H}$ type $\pi-\pi$ stacking on electronic properties of PDI aggregates (Top of Fig. 2b). The $d$ spacing of $\pi-\pi$ stacking for PDI molecules was set to be $0.34 \mathrm{~nm}$ according to XRD analysis above (Bottom of Fig. 2a). Vacuum energy level calibration method was applied to calculate the absolute energy level position of HOMO and LUMO of PDI aggregates. The ionic positions of the starting structures in the stack were optimized by the projector augmented wave (PAW) method with the Perdew-Burke-Ernzerhof including dispersion (PBE-D) exchange correlation functional in the Vienna ab initio simulation package (VASP, version 5.3.2).[23, 24] The LUMO/HOMO levels of PDI monomer were calculated to be $-4.49 /-5.93 \mathrm{eV}$, yielding an energy gap of $\sim 1.44 \mathrm{eV}$ (Table S2), which was much smaller than the experimental value $(2.25 \mathrm{eV})$.[30] The underestimation of band gap was attributed to the deficiency of DFT in the band gap calculation. Upon $\pi-\pi$ stacking, the calculated bandgap of PDI decreased obviously, and the absolute energy levels of HOMO and LUMO were both lowered (Fig. 2a, Table S2). Contrary to the isolated perylene diimide molecules with discrete energy levels, semiconductor-like continuous energy levels were formed upon $\pi$ - $\pi$ stacking (Fig. 2c).

The influence of H-type $\pi$ - $\pi$ stacking on the electronic structure was further confirmed by experimental results. The absolute energy levels of bulk PDI and nano PDI were evaluated by Mott-Schottky plots, UV-vis diffuse reflection spectra (DRS) 
and X-ray photoemission spectroscopy (XPS) valence band spectra measurements. The bottom of conduction band for bulk PDI and nano PDI can be estimated by the MottSchottky plots. As shown in Fig. 2d, the flat band potential $\left(E_{f b}\right)$ for bulk PDI and PDI nanofibers were $-0.40 \mathrm{~V},-0.21 \mathrm{~V}$ vs. SCE $(\mathrm{pH}=7)$, respectively. For n-type semiconductors, the bottom of conduction band (CB) is $0.2 \mathrm{~V}$ higher than that of Fermi level. Therefore, the CB of bulk PDI and PDI at normal hydrogen electrode (NHE, $\mathrm{pH}=7$ ) can be calculated using the following equation:

$$
\mathrm{E}_{\mathrm{CB}}(\mathrm{NHE}, \mathrm{pH}=7)=\mathrm{E}_{\mathrm{fb}}(\mathrm{SCE}, \mathrm{pH}=7)+0.24-0.2
$$

The results reveal that the CB position of bulk PDI and nano PDI were about -0.36 $\mathrm{V}$ and -0.17 vs. NHE (Fig. 2e). According to the band gap (1.74 eV and $1.69 \mathrm{eV})$, the valence band (VB) position of bulk PDI and nano PDI were estimated to be +1.38 and $+1.52 \mathrm{~V}$ vs. NHE. The schematic diagram of energy level of bulk PDI and nano PDI were summarized in Fig. 2f. The CB/VB of bulk PDI and nano PDI were evaluated to be $-0.36 /+1.38 \mathrm{~V}$ and $-0.17 /+1.52 \mathrm{~V}$. It is clear that the VB position of nano PDI was deeper than that of bulk PDI. This result can be further confirmed by X-ray photoemission spectroscopy (XPS) valence band spectra as shown in Fig. 2e. The deeper VB position means stronger oxidation ability of holes for photooxidation.

By incorporating theoretical and experimental methods, we demonstrated that the more favourable H-type $\pi$ - $\pi$ stacking in nano PDI leads to a narrowing of band gap, and lowers both VB and CB levels. Narrow bandgap (1.69 eV) facilitates induced charge carriers under visible light. And the deeper VB position $(+1.52 \mathrm{~V})$ leads to stronger oxidation ability of holes for photooxidation.

\subsection{Photooxidation performance of PDI nanofibers}

As a traditional antennae chromophore, PDIs were used to work with the central catalysts (especially for metal catalyst center) to construct water splitting system.[17, 18] In our work, PDI nanofibers can be used as a visible-light active photocatalyst without the apparent need for an added metal co-catalysts. Energy dispersive X-ray (EDX) spectroscopy analysis (Fig. S6) and EDX mapping (Fig. S7) measurements were 
performed to investigate the purity of PDI nanofibers, and the results confirm that the PDI nanofibers sample was without doubt pure. These results indicate that such pure supramolecular organic photocatalyst can harness visible light to split water and decompose phenol without the assistant of co-catalyst.

Organic pollutions decomposition experiments were carried out under visible light. In view of photosensitization of organic dyes, we choose phenol as the main model pollutant due to its stability under visible light irradiation without catalyst. What's more, dark adsorption experiment of phenol exhibits that there was no obviously adsorption distinction over the as-prepared photocatalysts (Fig. S8). For comparison purposes, the performance of bulk PDI, unmodified perylene diimide (PTCDI) and $\mathrm{g}-\mathrm{C}_{3} \mathrm{~N}_{4}$ (typical polymeric photocatalyst), under the same experimental conditions, were also shown (Fig. 3a). It was found that the process of phenol decomposition over PDI nanofibers was obviously quicker than that of bulk PDI, PTCDI and $g-\mathrm{C}_{3} \mathrm{~N}_{4}$. The apparent reaction rate was estimated to be as high as $0.129 \mathrm{~h}^{-1}$, which was about 20.2, 10.7 and 12.8 times higher than that of bulk PDI, PTCDI and $g-\mathrm{C}_{3} \mathrm{~N}_{4}$. The typical HPLC spectrum of phenol degradation of nano PDI was shown in Figure S9. The peaks appeared at low retention time can be attributed to intermediate products of phenol oxidation including hydroquinone (HQ), p-benzoquinone (p-BQ) and catechol. Furthermore, the total organic carbon (TOC) measurements were also carried out and the results further confirm the strong photooxidative ability of nano PDI (Fig. 3b). What's more, PDI nanofibers were also proved to be photoactive in the degradation of other common organic pollutions, such as methylene blue/MB (cationic dye), methyl orange/MO (anionic dyes) and bisphenol A/BPA (phenolic compounds) (Fig. S10).

The strong photooxidative ability of such supramolecular photocatalyst can be further certified by the water oxidation experiments. The photocatalytic oxygen evolution tests were carried out under visible light irradiation. Silver nitrate solution $(0.01 \mathrm{M})$ was elected as an electron acceptor. Simliarly, nano PDI exhibits more superior photocatalytic activities, which was about 19.8, 10.2 and 4.7 times higher than that of bulk PDI, PTCDI and $g-\mathrm{C}_{3} \mathrm{~N}_{4}$ (Fig. 3c). Note that the photocatalytic activity of bulk PDI was quite poor both in oxygen evolution and phenol degradation. The enhanced photocatalytic activities of PDI nanofibers were attributed to higher order $\pi$ - 
$\pi$ stacking and nanoctystalline.

More interestingly, such supramolecular photocatalyst exhibits broad spectral response owing to its narrow banggap (Fig. 3d). The trends of oxygen evolution rates of PDI nanofibers match well with its optical absorption. The quantum efficiency at the wavelength of $600 \pm 15 \mathrm{~nm}$ was estimated to be at least $0.56 \%$. Water oxidation reaction can be conducted even in near-infrared region $(\sim 700 \mathrm{~nm})$. Broad spectral response was of importance to the utilization of solar energy.

Due to the introduction of terminal carboxy group, the photocatalytic activity and stability of nano PDI were dependent on $\mathrm{pH}$ value of reaction solution. As shown in Fig. 4a, nano PDI exhibits superior photodegradation performance at neutral $(\mathrm{pH}=7)$ and acidic conditions ( $\mathrm{pH}=3$ ), whereas the photocatalytic activity at alkaline solution $(\mathrm{pH}=9)$ was quite poor. The lose of activity at alkaline solution can be attributed to the depolymerization of nano PDI owing to the deprotonation of PDI molecules. At $\mathrm{pH}=9$, the intermolecular hydrogen bonding between neighbouring carboxy group was destoryed. As a result, nano PDI depolymerized into molecular PDI. Compared to the supramolecular PDI blocks, PDI molecule shows much lower photocatalytic activity. This result reveals that the effective photocatalytic activity can be observed only when the self-assembly of PDI occurred, which will be further discussed as follows.

Furthermore, we found that nano PDI shows different stability at neutral and acidic conditions. The photocatalytic activity does not decrease at $\mathrm{pH}=3$ even after five cycles, whereas appreciable loss of activity can be found at $\mathrm{pH}=7$ (Fig. 4b). Meanwhile, investigation on the morphology of nano PDI shows that the morphology was maintain nanofibers at $\mathrm{pH}=3$ (Fig. 4c). In constrast, only nanopartilces can be found at $\mathrm{pH}=7$ after five five cycles (Fig. 4d). Interestingly, no differences have been found in FT-IR spectra of the nano PDI before and after catalysis either at $\mathrm{pH}=3$ or 7 (Fig. 4e), indicating that the molecule structure of PDI does not change during photocatalytic reaction. Fig. $4 \mathrm{f}$ shows the UV-vis spectra of the suspension solution of nano PDI before and after catalysis at $\mathrm{pH}=7$. It was clear that a new broad band emerged at 550-650 nm after catalysis, which can be belonged to J-type $\pi-\pi$ stacking of PDI.[31] PDI supramolecular blocks with J-type $\pi-\pi$ stacking structure was usually used as fluorescent materilas.[31-33] The recombination rate of photo-induced carrier in J-type 
aggregates was much higher than that of H-type aggregates, which may have side effects to the photocatalysis. The stability of nano PDI in acidic conditions may be attributed to strong hydrogen bonding interaction between carboxy group, which can stablize the H-type $\pi-\pi$ stacking of PDI. These results attest that shuch PDI supramolecular nanofibres were very stable photocatalysts in acidic conditions.

\subsection{Photocatalytic mechanism of PDI nanofibers}

The photocurrent tests were carried out without bias under visible light ( $>420 \mathrm{~nm})$. As shown in Fig. 5a, PDI monomer can hardly generate any photocurrent, while all of bulk PDI, nano PDI can exhibit photocurrent response under visible light. These results reveal that only when the self-assembly of PDI occurred, the effective photocarriers separation can be observed. What's more, the current intensity of nano PDI was much larger than that of bulk PDI. The enhanced photocurrent indicates a higher separation efficiency of photocarriers, which was attributed to the nanoctystalline and the higher order $\pi-\pi$ stacking in nano PDI. It was found that the internal electric field caused by carboxyl side chain of PDI molecule also plays crucial role in separation of photocarriers and photocatalytic activity. To further investigate the role of carboxyl side chain, alkyl side chain substituted PDI supramolecular (defined as PDI- $\mathrm{CH}_{3}$, detail synthesis information was shown in ESI) nanofibers were prepared (Fig. 5b). The photocurrent responses of PDI supramolecular nanofibers with and without carboxyl were shown in Fig. 5c. The results reveal that the photocurrent response decreases obviously when the carboxyl was removed. Moreover, PDI-CH 3 supramolecular nanofibers cannot split water for oxygen evolution under visible light (Fig. S12). Compared to the alkyl side chain, carboxyl group was more active for charge transport. The polar structure of PDI was shown in top of Fig. 5d. Because of the high electron affinity of perylene core, the intramolecular polarization was constructed from perylene core to imide substituents. The intramolecular polarization of PDI molecule contributes to the formation of internal electric field in nano PDI, which in turn benefits the separation and migration of photogenerated carriers. Under irradiation, the photogenerated electrons tend to remain in the core of PDI and transport through the direction of quasi-one-dimensional $\pi-\pi$ stacking, while holes prefer to migrate outside 
(Bottom of Fig. 5d).

To detect the active species of nano PDI during photocatalysis, the electron paramagnetic resonance (ESR) technique and trapping experiments were carried out. The superoxide radical $(\bullet \mathrm{OH})$ and hydroxyl radical $\left(\cdot \mathrm{O}_{2}^{-}\right)$can be detected by ESR technique using different solution. In the solution of DMSO, a noticeable signal of quartet can be observed under visible light irradiation, indicating that existence of $\bullet \mathrm{O}_{2}{ }^{-}$ (Fig. 6a). In contrast, no signal can be observed both with/without irradiation in water, suggesting that there was no $\bullet \mathrm{OH}$ in this supramolecular system (Fig. 6b). Such results can be further confirmed by trapping experiments. Fig. $6 \mathrm{c}$ shows the photodegradation tests of phenol with the addition of hole scavenger (potassium iodide, KI),[34] superoxides radical scavenger (p-benzoquinone, p-BQ)[35] and hydroxyl radical scavenger (tert-Butanol, t-BuOH)[34] under visible light irradiation, respectively. The addition of $\mathrm{t}-\mathrm{BuOH}$ has no effect on the photocatalytic degradation of phenol. On the contrary, the photocatalytic activity reduces drastically with the addition of KI and p$\mathrm{BQ}$, indicating that the hole $\left(\mathrm{h}^{+}\right)$and superoxide radical $\left(\cdot \mathrm{O}_{2}^{-}\right)$are the main oxidative species in photocatalytic process of PDI nanofibers.

Based on the discussion above, a possible photocatalytic mechanism of nano PDI was proposed as shown in Fig. 7. Firstly, organic semiconducting electronic structure was constructed through the self-assembly of PDI molecules. The H-type $\pi$ - $\pi$ stacking in PDI nanofibers leads to a great narrowing of band gap, and moves down both HOMO (VB) and LUMO (CB) levels. As a result, the electrons and holes can be generated in conduction and valence band respectively under near-infrared spectral irradiation owing to its narrow band gap $(1.69 \mathrm{eV})$. Deep valence band position $(+1.52 \mathrm{eV})$ facilitates holes to overcome the large overpotential of oxygen evolution (Fig. 7a). Secondly, benefit to the terminal carboxy group, well-defined H-type $\pi-\pi$ stacking can be formed in supramolecular nanofibers. Long-range electrons delocalization in $\pi-\pi$ stacking great promotes the migration efficiency of photo-induced charge carriers. Meanwhile, the internal electric field formed between perylene core and carboxylic substituents enhanced the separation efficiency of charge carriers, which was responsible for the enhancement of photocatalytic performance (Fig. 7b). 


\section{Conclusion}

In conclusion, an one-dimensional supramolecular nanofibers photocatalyst based on carboxy-substituent perylene diimide have been developed. Such purely organic PDI supramolecular nanofibers exhibit superior photocatalytic activity both in organic pollutions degradation and water oxidation under visible light without assistance of cocatalyst. Most importantly, these photocatalysts were demonstrated to be very stable with cycling at acidic condition, which crucial for industrial applications. Unique onedimension $\pi$-conjugated delocalization and internal electric field in supramolecular nanofibers result in the highly efficient excitation, separation and migration of carriers, which was demonstrated to be the key factor to the photocatalysis process. The catalyst has the advantages of sufficiently efficient, robust, earth-abundant and broad spectral response. The present results may offer a new vista for the development of supramolecular organic semiconductor in environmental protection and water splitting.

\section{Acknowledge}

This work was partly supported by National Basic Research Program of China (973 Program) (2013CB632403 and 2015CB655002) and National Science Foundation of China (21437003 and 21290190). Computational resources were provided by Tsinghua National Laboratory for Information Science and Technology of China.

\section{Appendix A. Supplementary data}

Supplementary data associated with this article can be found in the online version.

\section{References}

[1] A. Kudo and Y. Miseki, Chem. Soc. Rev. 38 (2009) 253-278.

[2] T. Zhang and W. Lin, Chem. Soc. Rev. 43 (2014) 5982-5993.

[3] X. Wang, K. Maeda, A. Thomas, K. Takanabe, G. Xin, J. M. Carlsson, K. Domen and M. Antonietti, Nature Mater. 8 (2009) 76-80.

[4] S. Ghosh, N. A. Kouamé, L. Ramos, S. Remita, A. Dazzi, A. Deniset-Besseau, P. Beaunier, F. Goubard, P.-H. Aubert and H. Remita, Nature Mater. 14 (2015) 505-511.

[5] R. S. Sprick, J.-X. Jiang, B. Bonillo, S. Ren, T. Ratvijitvech, P. Guiglion, M. A. Zwijnenburg, D. J. Adams and A. I. Cooper, J. Am. Chem. Soc. 137 (2015) 3265-3270.

[6] M. G. Schwab, M. Hamburger, X. Feng, J. Shu, H. W. Spiess, X. Wang, M. Antonietti and K. Müllen, Chem. Commun. 46 (2010) 8932-8934.

[7] J. Tang, J. R. Durrant and D. R. Klug, J. Am. Chem. Soc. 130 (2008) 13885-13891.

[8] C. Ego, D. Marsitzky, S. Becker, J. Zhang, A. C. Grimsdale, K. Müllen, J. D. MacKenzie, C. Silva and R. H. Friend, J. Am. Chem. Soc. 125 (2003) 437-443. 
[9] L. Zang, R. Liu, M. W. Holman, K. T. Nguyen and D. M. Adams, J. Am. Chem. Soc. 124 (2002) 10640-10641.

[10] M. Sauer, Angew. Chem. 42 (2003) 1790-1793.

[11] Y. Wen, Y. Liu, C. a. Di, Y. Wang, X. Sun, Y. Guo, J. Zheng, W. Wu, S. Ye and G. Yu, Adv. Mater. 21 (2009) 1631-1635.

[12] A. S. Molinari, H. Alves, Z. Chen, A. Facchetti and A. F. Morpurgo, J. Am. Chem. Soc. 131 (2009) 2462-2463.

[13] L. Schmidt-Mende, A. Fechtenkötter, K. Müllen, E. Moons, R. H. Friend and J. MacKenzie, Science 293 (2001) 1119-1122.

[14] R. Singh, E. Giussani, M. M. Mróz, F. Di Fonzo, D. Fazzi, J. Cabanillas-González, L. Oldridge, N. Vaenas, A. G. Kontos and P. Falaras, Org. Electron. 15 (2014) 1347-1361.

[15] F. Würthner, Chem. Commun. (2004) 1564-1579.

[16] A. S. Weingarten, R. V. Kazantsev, L. C. Palmer, M. McClendon, A. R. Koltonow, A. P. Samuel, D.

J. Kiebala, M. R. Wasielewski and S. I. Stupp, Nature Chem. 6 (2014) 964-970.

[17] V. Kunz, V. Stepanenko and F. Würthner, Chem. Commun. 51 (2015) 290-293.

[18] S. Chen, D. L. Jacobs, J. Xu, Y. Li, C. Wang and L. Zang, RSC Adv. 4 (2014) 48486-48491.

[19] S. Chen, C. Wang, B. R. Bunes, Y. Li, C. Wang and L. Zang, Appl. Catal. A 498 (2015) 63-68.

[20] D. Liu, J. Wang, X. Bai, R. Zong, Y. Zhu, Adv. Mater. (2016) 10.1002/adma.201601168.

[21] C. Xue, M. Chen and S. Jin, Polymer 49 (2008) 5314-5321.

[22] A. Datar, K. Balakrishnan and L. Zang, Chem. Commun. 49 (2013) 6894-6896.

[23] P. E. Blöchl, Physical Review B 50 (1994) 17953.

[24] S. Grimme, J. Comput. Chem. 27 (2006) 1787-1799.

[25] Z. Chen, V. Stepanenko, V. Dehm, P. Prins, L. D. Siebbeles, J. Seibt, P. Marquetand, V. Engel and F. Wuerthner, Chem.-Eur. J. 13 (2007) 436-449.

[26] M. Angelella, C. Wang and M. J. Tauber, J. Phys. Chem. A, 2013, 117 (2009) 9196-9204.

[27] P. Yan, A. Chowdhury, M. W. Holman and D. M. Adams, J. Phys. Chem. B 109 (2005) 724-730.

[28] K. Balakrishnan, A. Datar, T. Naddo, J. Huang, R. Oitker, M. Yen, J. Zhao and L. Zang, J. Am. Chem. Soc. 128 (2006) 7390-7398.

[29] R. F. Fink, J. Seibt, V. Engel, M. Renz, M. Kaupp, S. Lochbrunner, H.-M. Zhao, J. Pfister, F. Würthner and B. Engels, J. Am. Chem. Soc. 130 (2008) 12858-12859.

[30] M. C. R. Delgado, E.-G. Kim, D. t. A. d. S. Filho and J.-L. Bredas, J. Am. Chem. Soc. 132 (2010) 3375-3387.

[31] F. Würthner, C. Thalacker, S. Diele and C. Tschierske, Chem.-Eur. J. 10 (2001) 2245-2253.

[32] Z. Chen, V. Stepanenko, V. Dehm, P. Prins, and F. Würthner, Chem.-Eur. J. 13 (2007) 436-449.

[33] H. Lin, R. Camacho, Y. Tian, T. E. Kaiser, F. Würthner and I. G. Scheblykin, Nano Lett. 10 (2010) 620-626.

[34] G. Dong, W. Ho and L. Zhang, Appl. Catal. B 168 (2015) 490-496.

[35] E. M. Rodríguez, G. Márquez, M. Tena, P. M. Álvarez and F. J. Beltrán, Appl. Catal. B 178 (2015) 44-53.

\section{Figure captions}

Figure 1. (a, b) Transmission electron micrograph (TEM) images of bulk PDI (a) and nano PDI (b). c, Schematic diagram showing the self-assembling process drived by strong $\pi-\pi$ stacking and hydrogen bonding. d, UV-vis absorption spectra of PDI monomer soultion and nano PDI suspension. e, Experimental XRD patterns of the bulk PDI and nano PDI powders. f, The UV-vis diffuse reflection spectra (DRS) of bulk PDI and nano PDI powders.

Figure 2. a, Top: Schematic diagram showing the frontier molecular orbitals of PDI molecule; Bottom: d-spacing of $\pi-\pi$ stacking. $\mathbf{b}$, Energy diagram representing the 
theoretically evaluated HOMO and LUMO levels of $\pi-\pi$ stacked PDI aggregates (comprising $n=1,2,3$ and infinite monomers). c, Projected DOS of PDI aggregates with infinite degree of $\pi-\pi$ stacking. d, Mott-Schottky plots of bulk PDI and nano PDI samples; counter electrode: Pt. electrolyte: $0.1 \mathrm{M} \mathrm{Na}_{2} \mathrm{SO}_{4}$, frequency: $100 \mathrm{kHz}$; e, Xray photoemission spectroscopy (XPS) valence band spectrum of nano PDI. f, Diagram representing experimentally evaluated HOMO (VB) and LUMO (CB) levels of bulk PDI and nano PDI.

Figure 3. a, Photodegradation curves for phenol (5 ppm) over bulk PDI, nano PDI, PTCDI and $\mathrm{g}_{-} \mathrm{C}_{3} \mathrm{~N}_{4}$. b, Total organic carbon (TOC) removal rate for phenol (10 ppm) over nano PDI under visible light. c, Oxygen evolution from water by bulk PDI, nano PDI, PTCDI and $g-\mathrm{C}_{3} \mathrm{~N}_{4}$ in the presence of an electron acceptor (10 $\mathrm{mM}$ silver nitrate). d, Wavelength-dependent oxygen evolution by nano PDI (Band pass filter, 500 \pm 15 , $550 \pm 15, \ldots, 700 \pm 15 \mathrm{~nm})$.

Figure 4. a, Photodegradation curves for phenol (5 ppm) over nano PDI at different $\mathrm{pH}=3,7$ and 9. b, Degradation rate constants $\mathrm{k}$ of phenol at $\mathrm{pH}=3$ and 7 during recycling tests. (c, d) TEM images of the nano PDI after degradation of phenol at $\mathrm{pH}=3$ (c) and $\mathrm{pH}=7$ (d) under visible light irradiation. e, FT-IR spectra of the nano PDI before and after catalysis at $\mathrm{pH}=3$ and 7. f, $\mathrm{UV}$-vis spectra of the suspension solution of nano PDI before (red dotted line) and after (black solid line) catalysis at $\mathrm{pH}=7$.

Figure 5. a, Photocurrents of PDI monomer, bulk PDI and nano PDI without bias under visible light (>420 nm). b, Transmission electron micrograph (TEM) image of alkyl side chain substituted PDI (defined as PDI- $\mathrm{CH}_{3}$ ) supramolecular nanofibers. c, Photocurrents of nano PDI and PDI-CH 3 nanofibers. d, Top: Polarity structure of PDI molecule; Bottom: Schematic diagram showing the migration of carriers in quasi-onedimensional H-type $\pi$ - $\pi$ stacking model.

Figure 6. (a, b) Electron paramagnetic resonance (ESR) spectra of nano PDI in DMSO solvents (a) and water (b). c, Plots of photogenerated carriers trapping in the system of photodegradation of phenol by nano PDI.

Figure 7. a, Diagram representing electronic properties of PDI nanofibers. b, Mechanism of charges separation and photocatalytic reaction of PDI nanofibers under visible light. 

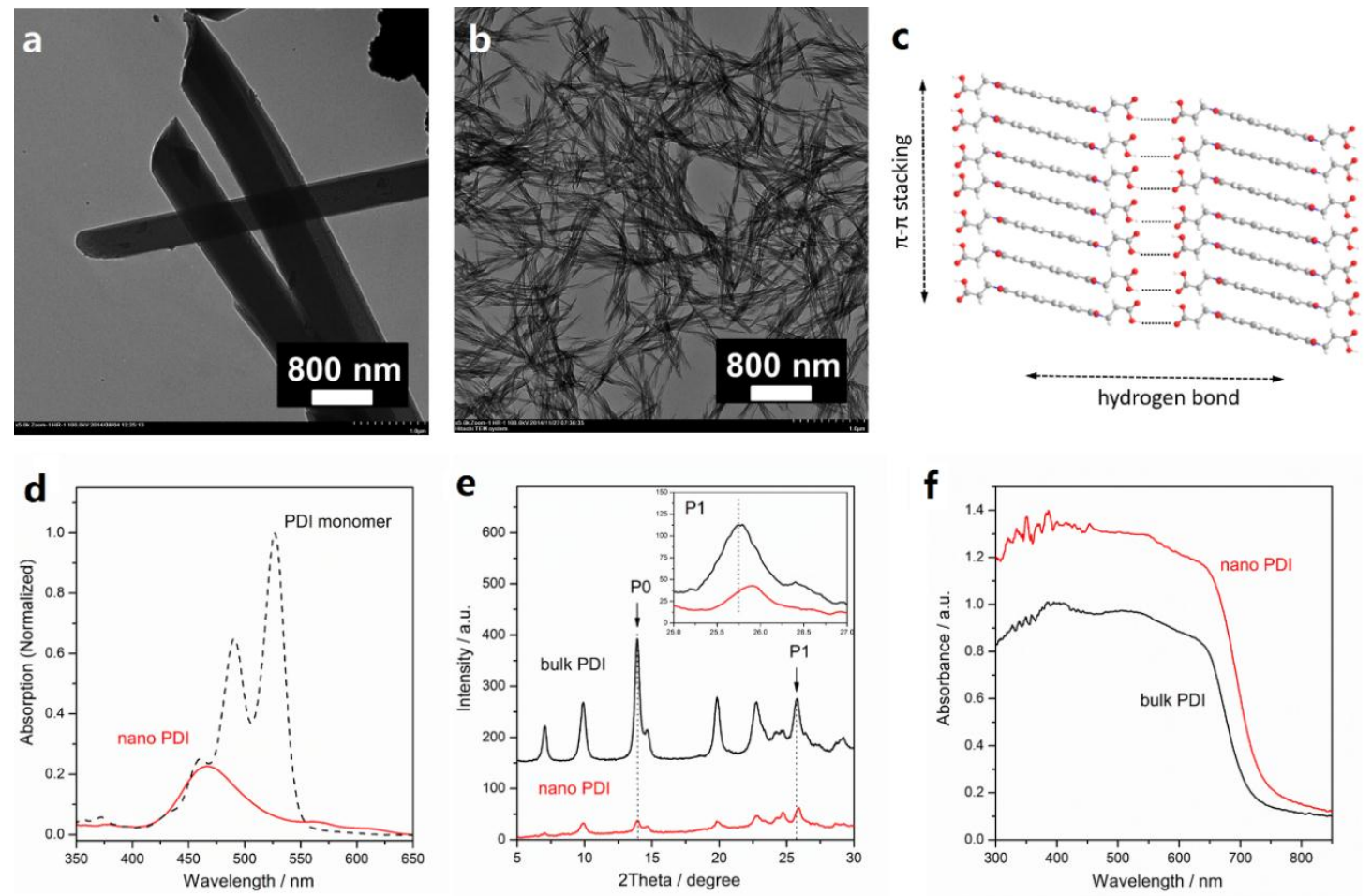

Figure 1. (a, b) Transmission electron micrograph (TEM) images of bulk PDI (a) and nano PDI (b). c, Schematic diagram showing the self-assembling process drived by strong $\pi-\pi$ stacking and hydrogen bonding. d, UV-vis absorption spectra of PDI monomer soultion and nano PDI suspension. e, Experimental XRD patterns of the bulk PDI and nano PDI powders. f, The UV-vis diffuse reflection spectra (DRS) of bulk PDI and nano PDI powders. 

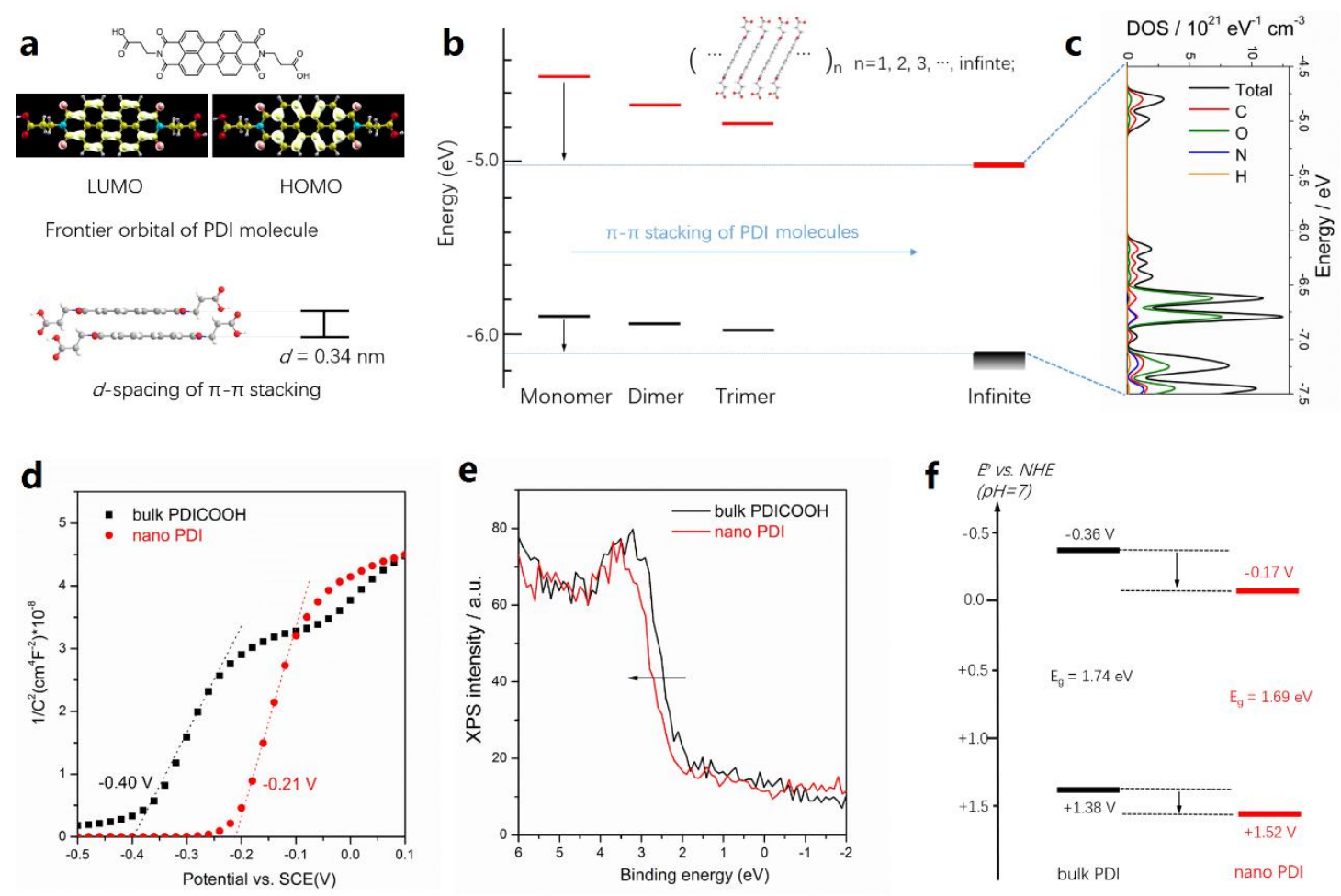

Figure 2. a, Top: Schematic diagram showing the frontier molecular orbitals of PDI molecule; Bottom: d-spacing of $\pi-\pi$ stacking. $\mathbf{b}$, Energy diagram representing the theoretically evaluated HOMO and LUMO levels of $\pi-\pi$ stacked PDI aggregates (comprising $\mathrm{n}=1,2,3$ and infinite monomers). c, Projected DOS of PDI aggregates with infinite degree of $\pi-\pi$ stacking. d, Mott-Schottky plots of bulk PDI and nano PDI samples; counter electrode: Pt. electrolyte: $0.1 \mathrm{M} \mathrm{Na}_{2} \mathrm{SO}_{4}$, frequency: $100 \mathrm{kHz}$; e, Xray photoemission spectroscopy (XPS) valence band spectrum of nano PDI. f, Diagram representing experimentally evaluated HOMO (VB) and LUMO (CB) levels of bulk PDI and nano PDI. 

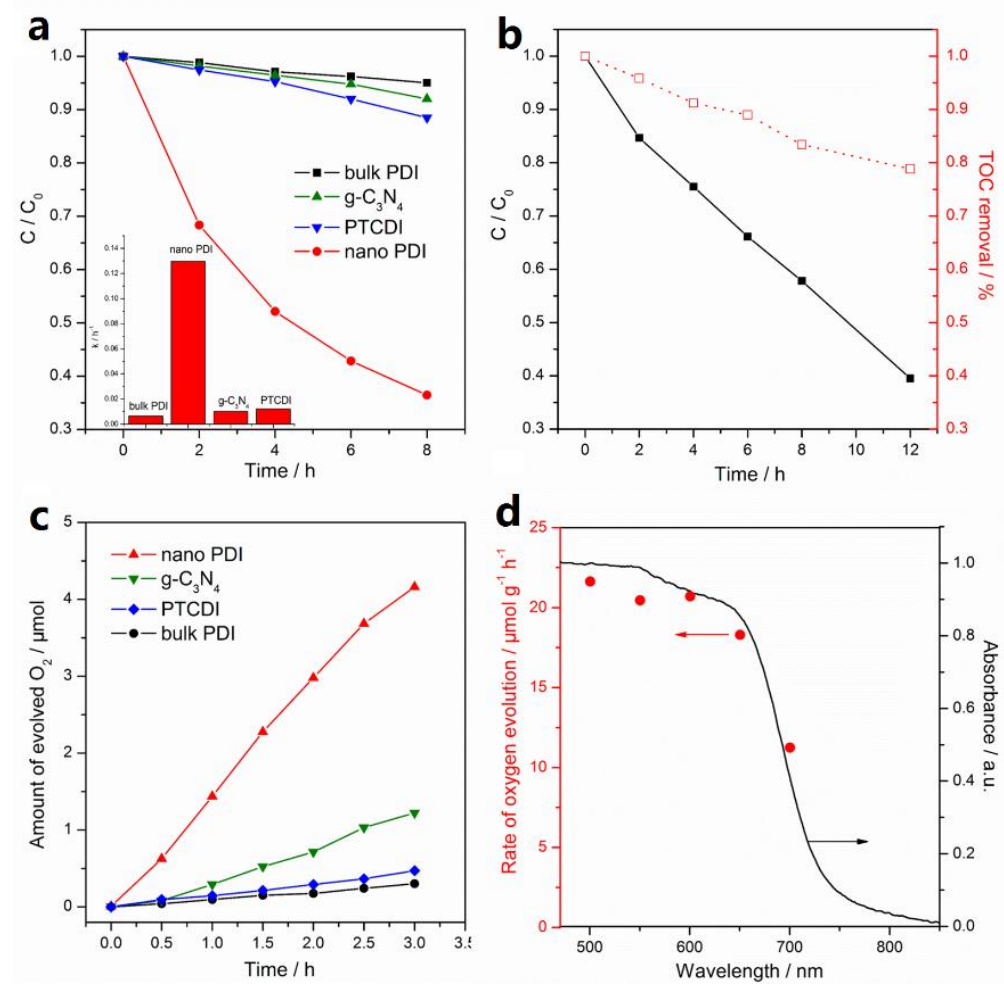

Figure 3. a, Photodegradation curves for phenol ( $5 \mathrm{ppm}$ ) over bulk PDI, nano PDI, PTCDI and $\mathrm{g}_{-} \mathrm{C}_{3} \mathrm{~N}_{4}$. b, Total organic carbon (TOC) removal rate for phenol (10 ppm) over nano PDI under visible light. c, Oxygen evolution from water by bulk PDI, nano PDI, PTCDI and $\mathrm{g}-\mathrm{C}_{3} \mathrm{~N}_{4}$ in the presence of an electron acceptor (10 $\mathrm{mM}$ silver nitrate). d, Wavelength-dependent oxygen evolution by nano PDI (Band pass filter, 500 \pm 15 , $550 \pm 15, \ldots, 700 \pm 15 \mathrm{~nm})$. 

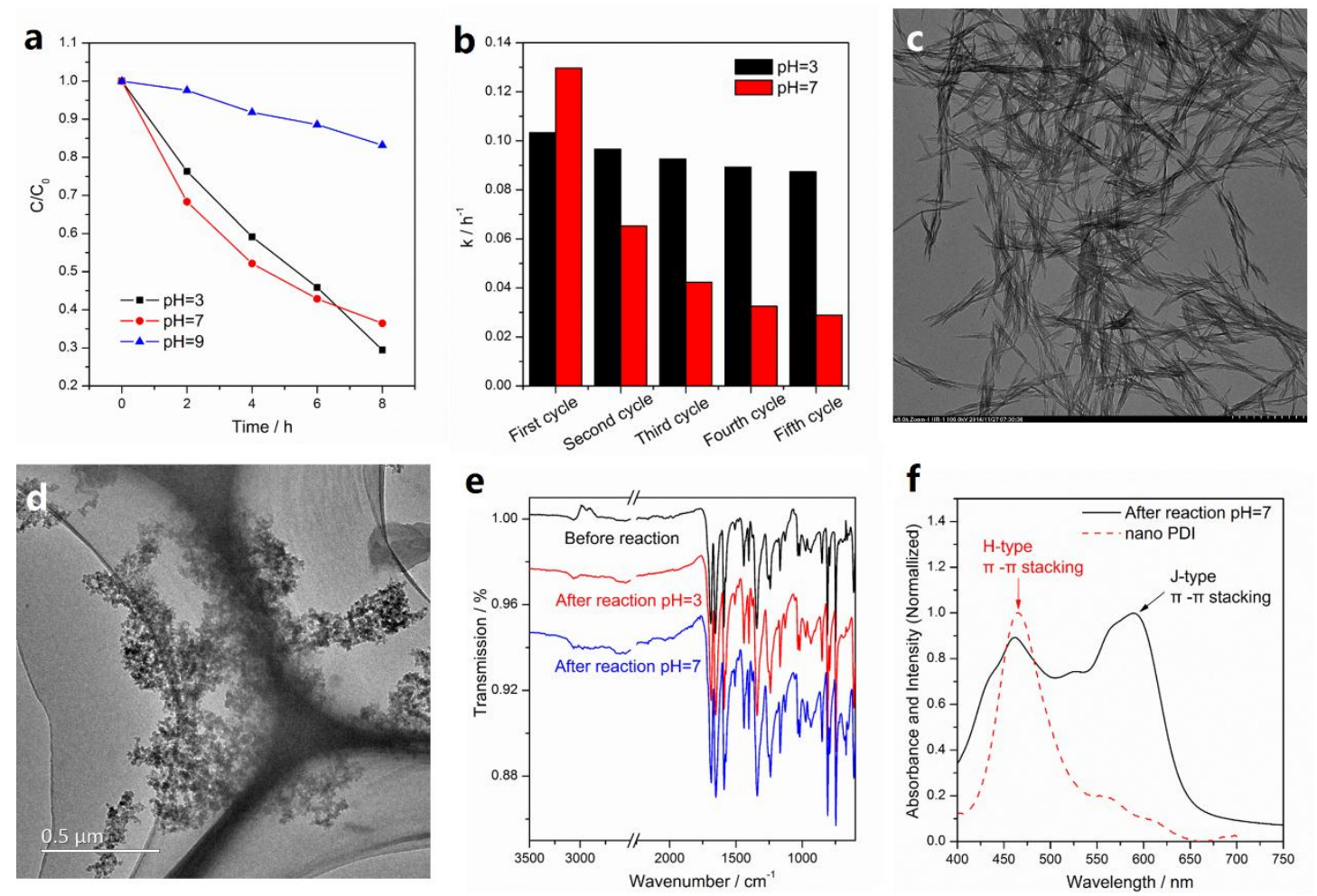

Figure 4. a, Photodegradation curves for phenol (5 ppm) over nano PDI at different $\mathrm{pH}=3,7$ and 9. b, Degradation rate constants $k$ of phenol at $\mathrm{pH}=3$ and 7 during recycling tests. (c, d) TEM images of the nano PDI after degradation of phenol at $\mathrm{pH}=3$ (c) and $\mathrm{pH}=7$ (d) under visible light irradiation. e, FT-IR spectra of the nano PDI before and after catalysis at $\mathrm{pH}=3$ and 7. f, $\mathrm{UV}$-vis spectra of the suspension solution of nano PDI before (red dotted line) and after (black solid line) catalysis at $\mathrm{pH}=7$. 

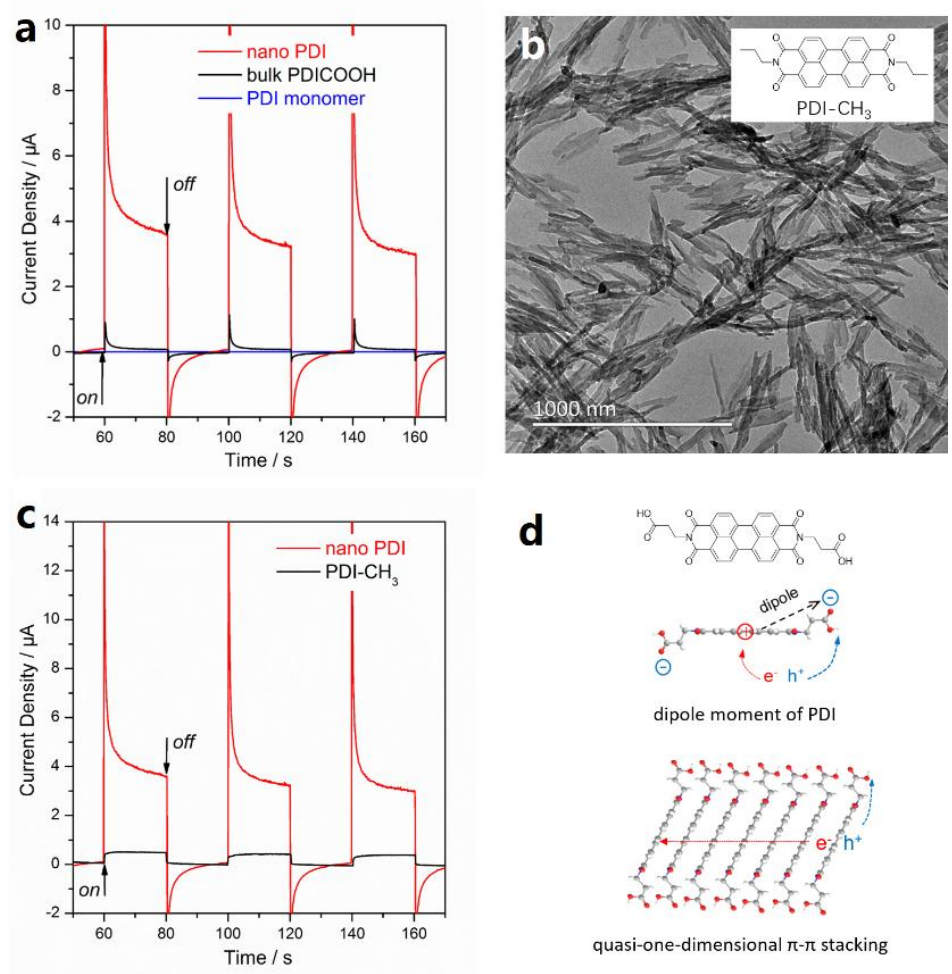

Figure 5. a, Photocurrents of PDI monomer, bulk PDI and nano PDI without bias under visible light (>420 nm). b, Transmission electron micrograph (TEM) image of alkyl side chain substituted PDI (defined as PDI-CH 3 ) supramolecular nanofibers. c, Photocurrents of nano PDI and PDI-CH 3 nanofibers. d, Top: Polarity structure of PDI molecule; Bottom: Schematic diagram showing the migration of carriers in quasi-onedimensional H-type $\pi$ - $\pi$ stacking model.
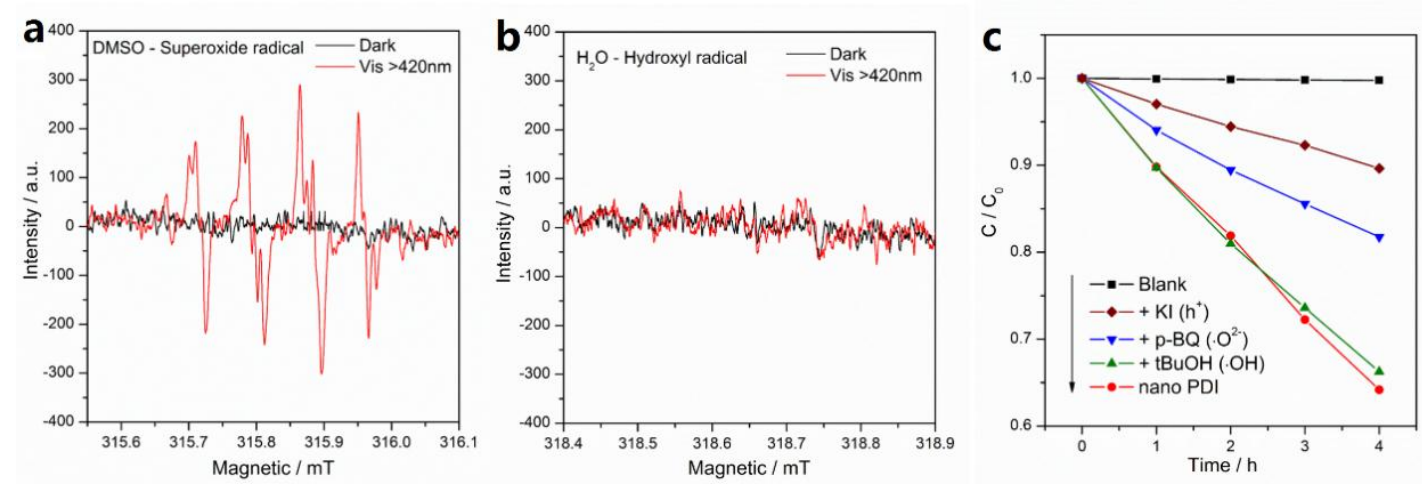

Figure 6. (a, b) Electron paramagnetic resonance (ESR) spectra of nano PDI in DMSO solvents (a) and water (b). c, Plots of photogenerated carriers trapping in the 
system of photodegradation of phenol by nano PDI.
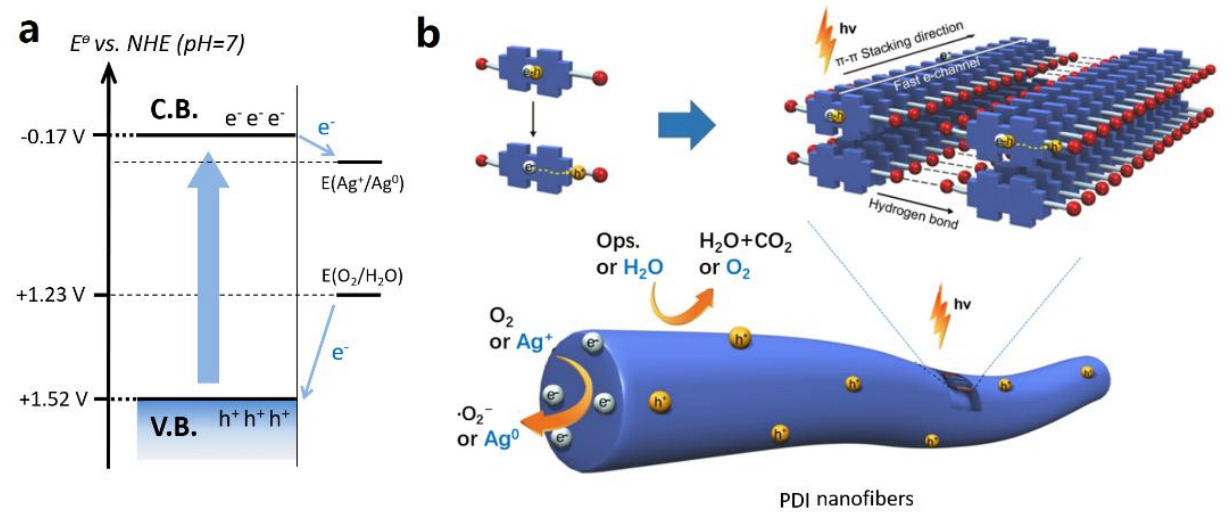

Figure 7. a, Diagram representing electronic properties of PDI nanofibers. b, Mechanism of charges separation and photocatalytic reaction of PDI nanofibers under visible light. 

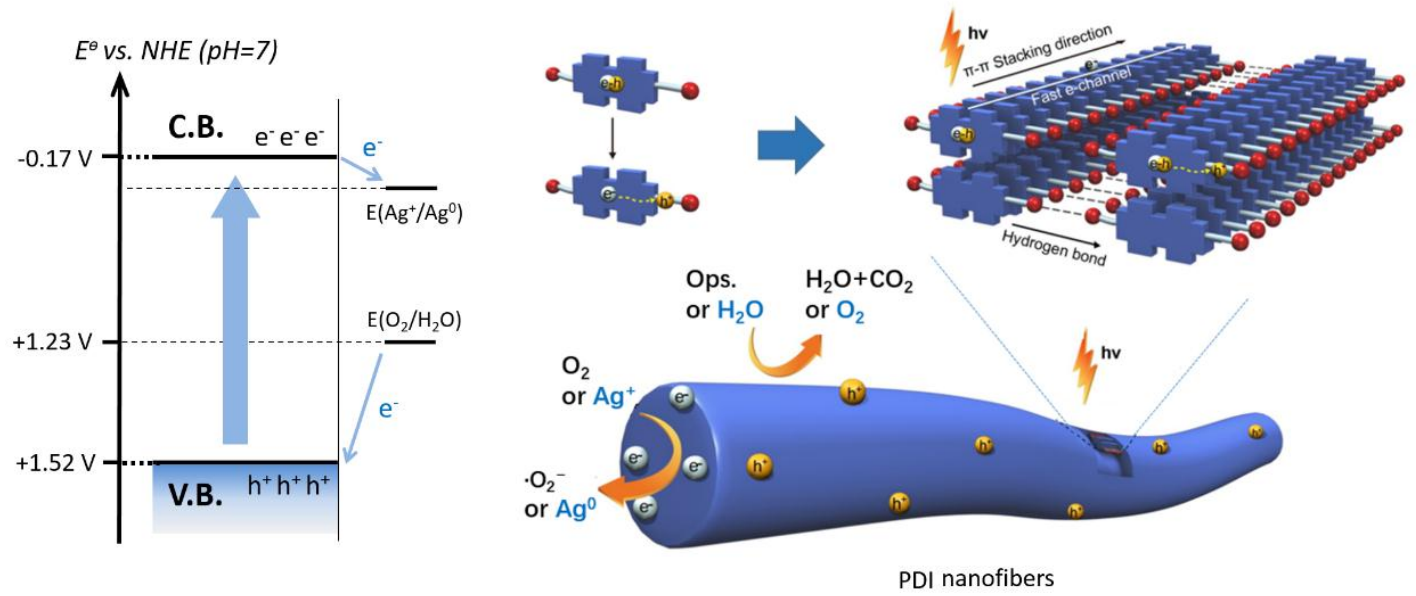